\title{
Características clínico-epidemiológicas de la estrongiloidiasis en pacientes portadores de co-morbilidades
}

\author{
Amanda Regueira Fernandes, Sebastián Romero, Paula Fernanda Alcântara de Souza Melo, \\ Paulo Sérgio Ramos Araújo, Oscar Bottasso, Abraham Rocha y Eduardo Brandão
}

\section{Clinical and epidemiological characteristics of strongyloidiasis in patients with comorbidities}

The strongyloidiasis is a parasitic disease that poses as a serious public health problem, mainly in tropical and subtropical countries. Over the years, some conditions, such as advances in corticosteroid treatment and immunosuppressive diseases, have improved not only the increase in cases of strongyloidiasis, but also the emergence of severe forms of the disease and / or deaths. For these reasons, the objective of this study is to make a critical analysis of the occurrence of strongyloidiasis in patients with comorbidities, describing clinical and epidemiological characteristics associated with these diseases that can highlight the importance of monitoring this parasitosis in most susceptible groups.

Key words: Strongyloidiasis; clinical features; epidemiology; comorbidities; Public Health.

Palabras clave: Estrongiloidiasis; manifestaciones clínicas; epidemiologia; co-morbilidades; Salud Pública.

\section{Introducción}

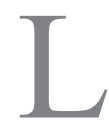

as geo-helmintiasis continúan siendo un importante problema de salud pública en países subdesarrolla$\operatorname{dos}^{1}$. Datos actuales estiman que aproximadamente dos billones de individuos en todo el mundo albergan por lo menos una especie de geo-helminto ${ }^{2}$. Dentro de estas parasitosis, algunas son más relevantes del punto de vista clínico-epidemiológico debido a su capacidad de producir formas complicadas de la enfermedad pudiendo evolucionar a la muerte, cuando no son tratadas adecuadamente, como por ejemplo, la esquistosomiasis y la estrongiloidiasis ${ }^{3,4}$.

Con relación a la estrongiloidiasis, la Organización Mundial de la Salud (OMS) estima que cerca de 370 millones de individuos están infectados en el mundo ${ }^{5}$; sin embargo, estos datos pueden estar subestimados debido al número de individuos no diagnosticados. En Brasil, esta parasitosis es considerada una enfermedad olvidada, de carácter endémico, y su análisis epidemiológico permitió identificar prevalencias de 5,3\% en el norte del país, 7,9\% en el nordeste, $6,6 \%$ en el centro oeste y 3,9 y $4,0 \%$ para las regiones sudeste y sur, respectivamente ${ }^{6}$.

Del punto de vista clínico, la estrongiloidiasis se caracteriza por presentar un amplio espectro de manifestaciones que varían desde la enfermedad asintomática hasta hiperinfección y estrongiloidiasis diseminada ${ }^{7}$. Según Gottardi la multiplicidad de presentaciones clínicas está relacionada a la inmunidad del hospedero.

Dentro de las manifestaciones agudas, los síntomas más observados son el eritema serpiginoso (producido por el contacto de la larva con la piel), la tos (similar al asma debido a la migración de la larva hacia los pulmones), dolor abdominal y/o diarrea ${ }^{8}$. Estos síntomas son inespecíficos y habitualmente cursan sin establecerse un diagnóstico específico ${ }^{9}$.

La estrongiloidiasis crónica es, en general, asintomática; sin embargo, pueden aparecer síntomas pulmonares y gastrointestinales producidos por la gran multiplicación y migración de larvas infectantes (síndrome de hiperinfección), especialmente en casos de inmunosupresión 9 .

La estrongiloidiasis diseminada ha sido descrita como una forma letal y está caracterizada por la afectación de múltiples órganos y sistemas, además del pulmonar y el gastrointestinal. La mortalidad puede llegar a $87 \%$ y está frecuentemente asociada a infección bacteriana secundaria, como por ejemplo, meningitis por Streptococcus bovis $^{10}$. Otras manifestaciones, menos frecuentes, pueden ser petequias o púrpuras, cefalea, convulsión y coma ${ }^{7}$.

En la mayoría de los casos de estrongiloidiasis grave, los eosinófilos periféricos son típicamente infrecuentes o más aún, están ausentes. Éstos son capaces de destruir a los helmintos a través de citotoxicidad celular dependiente de anticuerpos y junto a los mastocitos participan en la defensa contra las larvas que atraviesan los tejidos. La interacción del parásito con complejos IgE y mastocitos, conduce a la degranulación de los mismos y destrucción del parásito ${ }^{11,12}$.

A lo largo de los años, se han identificado factores de riesgo asociados al desarrollo de formas graves de
Fundação Oswaldo Cruz, Pernambuco, Brasil. Serviço de Referência Nacional em Filarioses, Departamento de Parasitología.

Centro de Pesquisas Aggeu Magalhães.

Universidad Nacional de Rosario, Rosario, Argentina. Facultad de Ciencias Médicas. Hospital Otávio de Freitas, Pernambuco, Brasil.

Secretaria de Saúde do Estado de Pernambuco.

Los autores declaran que no hay conflicto de interés.

No hubo financiamiento para el presente estudio.

Recibido: 7 de marzo de 2016 Aceptado: 19 de diciembre de 2016

Correspondencia a:

Eduardo Brandão

brandaoe@cpqam.fiocruz.br 
la parasitosis. Fardet y cols., observaron una mayor frecuencia de parasitados por Strongyloides stercoralis en individuos alcohólicos, diabéticos, portadores del virus linfotrópico humano de células T del tipo 1 (HTLV-1), pacientes receptores de trasplantes o en corticoterapia ${ }^{13}$. Otros estudios realizados identificaron la ocurrencia de estrongiloidiasis en pacientes infectados por el virus de la inmunodeficiencia humana (VIH) y con neoplasias hematológicas, con prevalencias que variaban entre 5,5 a $12,0 \%{ }^{14,15}$ y 8,3 a $23 \%{ }^{16,17}$, respectivamente.

Dicho esto y en consideración que los trabajos relacionados a infección por $S$. stercoralis, en pacientes que presentan otras co-morbilidades, han sido aislados, el objetivo del presente estudio es describir las características clínico-epidemiológicas de las principales condiciones referidas por la literatura científica como factores de riesgo para estrongiloidiasis, promoviendo una actualización crítica sobre el tema.

\section{Estrongilloidiasis y trasplantes}

Con el aumento del número de trasplantes y el desarrollo de fármacos inmunosupresores más potentes, la frecuencia de rechazo ha disminuido, pero en contrapartida, los receptores de órganos se han tornado más susceptibles a infecciones oportunistas ${ }^{18}$. Éstas pueden ser resultado de una infección primaria adquirida, por exposición posttrasplante o por reactivación de una infección latente ${ }^{19}$. La estrongiloidiasis en pacientes que recibieron un trasplante tiene importancia debido a que el tratamiento inmunosupresor genera que sean más susceptibles a desarrollar la infección o sus formas graves en pacientes ya infectados, como por ejemplo, síndrome de hiperinfección (presencia de gran cantidad de parásitos en un mismo órgano, en general intestino) y la forma diseminada (afectación de múltiples órganos $)^{20}$.

La preocupación con la asociación de trasplantes y estrongiloidiasis ha sido mencionada en países desarrollados, principalmente por el hecho de que muchos pacientes provienen de América Latina, donde la endemia de $S$. stercoralis es importante ${ }^{21}$. Un estudio realizado en Brasil con 1.754 receptores de trasplantes identificó que $82(4,7 \%)$ de esos pacientes desarrollaron algún tipo de enfermedad endémica o infección oportunista; de estos, 21 pacientes poseían alguna enteroparasitosis, siendo 2,4\% de estas, correspondientes a infección por $S$. stercoralis ${ }^{22}$. Los síntomas más frecuentes que presentan estos pacientes son diarrea y alteraciones gastrointestinales y aparecen en un promedio de seis meses post-trasplante ${ }^{18,23,24}$. Otro estudio, realizado con individuos latinoamericanos candidatos a recibir un trasplante, identificó una positividad para el parásito de $6 \%{ }^{25}$.

Diversos estudios discuten la ocurrencia atípica de $S$. stercoralis en pacientes que han recibido un trasplante. A pesar de que el parásito adulto vive en el tracto gastrointestinal, se observaron larvas en la orina, así como en lesiones inflamatorias en los riñones ${ }^{18,26}$. También se han descrito casos de estrongiloidiasis post-trasplante de hígado ${ }^{27}$, de corazón ${ }^{28}$ y precursores hematopoyéticos ${ }^{29}$, siendo este último, el grupo con mayor riesgo de hiperinfección fatal por S. stercoralis ${ }^{8}$.

Debido a la posibilidad de hiperinfección y diseminación de $S$. stercoralis post-trasplante, es extremamente oportuno un diagnóstico preciso de esta helmintiasis para que el tratamiento efectivo sea realizado precozmente ${ }^{8}$.

\section{Estrongiloidiasis y etilismo}

Algunos estudios sugieren que el etilismo crónico es un importante factor de riesgo para el desarrollo de estrongiloidiasis ${ }^{30,31}$. Estudios realizados por Oliveira y cols., demostraron frecuencias de 33,3 y 5,5\% de estrongiloidiasis en pacientes alcohólicos y no alcohólicos, respectivamente $^{30}$. Marques y cols., evaluaron 263 pacientes consumidores crónicos de alcohol, identificando una tasa de infección de 20,5\% mientras en 590 individuos no alcohólicos, tan sólo 4,4\% resultaron positivos ${ }^{32}$. En 2016, Silva y cols., identificaron resultados semejantes a los presentados en la literatura médica, observando una frecuencia de estrongiloidiasis entre individuos alcohólicos de $23,5 \%$ y entre no consumidores de alcohol de $5,4 \%{ }^{33}$.

De acuerdo con Gaburri y cols., esta predisposición estaría asociada con la baja higiene, la alta exposición al patógeno, malnutrición, alteración de la barrera intestinal y/o en la respuesta inmune local ${ }^{34}$. Otro estudio, realizado por Zago-Gomes y cols., indica que la mayor frecuencia de S. stercoralis entre individuos alcohólicos podría ser explicada por la alteración del patrón de respuesta inmune producida por la ingestión abusiva de alcohol y/o por la alteración del metabolismo de los corticosteroides inducida por el etanol ${ }^{31}$.

Sobre la patogenia de la infección en individuos alcohólicos, estudios sugieren que la intoxicación alcohólica puede elevar las concentraciones de corticosteroides endógenos humanos que suprimen la función de las células $\mathrm{T}$ y aumentan la fecundidad y supervivencia de los parásitos. Altos niveles de cortisol estimulan la transformación de las larvas rabditoides en larvas filarioides, por la mimetización de los ecdisteroides del parásito adulto $^{31,35,36}$. Además, el consumo crónico de alcohol tiene un efecto tóxico sobre las proteínas contráctiles del músculo del intestino delgado y en la función vagal, causando la reducción del tránsito gastrointestinal que aumenta el riesgo de autoinfección ${ }^{37}$.

Los resultados presentados refuerzan el concepto que el etilismo crónico es un importante factor predisponente 
para estrongiloidíasis, siendo altamente recomendable que se investigue la infección por $S$. stercoralis en individuos alcohólicos, aun cuando no presenten síntomas sugerentes de la parasitosis, pudiendo, esta investigación, ser realizada a través de la búsqueda de larvas en muestras de heces o por la detección de anticuerpos específicos en el suero ${ }^{38}$.

\section{Estrongiloidiasis y diabetes mellitus}

La diabetes mellitus (DM) es un trastorno metabólico caracterizado por la elevación de las concentraciones de glucosa en la sangre, que puede ocurrir debido a una reducción en la secreción o en la acción de la hormona insulina ${ }^{39}$. Los individuos diabéticos son descritos por la literatura como pacientes que están altamente predispuestos al desarrollo de otras enfermedades, principalmente aquellas de origen infeccioso ${ }^{40,41}$.

La relación entre la estrongiloidiasis y la DM no está claramente dilucidada. Estudios realizados por Mendonça y cols., hacen énfasis en la importancia de la parasitosis en individuos diabéticos, debido a que, identificaron una alta frecuencia de infección por S. stercoralis $(23 \%$ de positividad entre individuos diabéticos, contra 7,1\% en el grupo control) y el surgimiento de formas graves de la parasitosis en estos pacientes ${ }^{40}$. Nazligul y cols., estudiando la frecuencia de parasitosis en la población diabética, encontraron resultados discordantes, siendo en su experiencia, la frecuencia de la infección más elevada entre pacientes no portadores de DM. Sin embargo, la baja incidencia observada en este estudio puede ser resultado de la mayor cantidad de exámenes y visitas al laboratorio a las que estos pacientes fueron sometidos ${ }^{41}$.

De acuerdo con Bessman y Sapico (1992), en individuos diabéticos, la diminución de la perfusión arterial, neuropatías y respuesta inmune suprimida, serían los factores que favorecen el aumento de la frecuencia y gravedad de las enfermedades infecciosas ${ }^{42}$. De cualquier forma, resultan necesarios nuevos estudios para aclarar si realmente existe una asociación y también cuáles serían los mecanismos patogénicos envueltos en este proceso.

\section{Estrongiloidiasis y cáncer gástrico}

Cerca de $65 \%$ de los pacientes diagnosticados con cáncer gástrico tienen más de 50 años. En Brasil, estos tumores aparecen en tercer lugar en la incidencia entre hombres y en quinto, entre las mujeres ${ }^{43}$. Estudios realizados por Machados y cols., señalan la ocurrencia de muchos casos de hiperinfección por S. stercoralis en pacientes con diversos tipos de cáncer ${ }^{44}$.

En 2008 fue publicado un estudio sobre 77 pacientes que presentaban diversos tipos de cáncer, todos en trata- miento quimioterápico, de los cuales, 33 pacientes poseían cáncer gástrico. Después de exámenes inmunológicos y parasitológicos, la frecuencia de diagnósticos positivos para S. stercoralis fue de $24,2 \%$ en los pacientes con cáncer gástrico contra $4,5 \%$ en el grupo control, constituido por portadores de otros tipos de cáncer ${ }^{44}$.

El número de resultados positivos para estrongiloidiasis en portadores de cáncer gástrico debe ser probablemente, resultado de la neutropenia, inducida por la quimioterapia ${ }^{45,46}$. Estos números pueden ser todavía mayores considerando la endemicidad del área estudiada ${ }^{44}$. Otra hipótesis que también podría explicar esta relación es el hecho de que el parásito habita en la mucosa donde libera sustancias que podrían inducir el desarrollo de neoplasias gastrointestinales ${ }^{44,47}$.

Debido al impacto de la estrongiloidiasis en pacientes con cáncer, algunos estudios preconizan la realización de exámenes dirigidos a identificar la presencia del parásito, principalmente en aquellos individuos que residen en áreas endémicas, antes y/o durante la instalación de una terapia antineoplásica e inmunosupresora. Esa conducta permitiría el diagnóstico y el tratamiento precoz de la infección, reduciendo el riesgo de que este paciente desarrolle formas graves de la enfermedad e incluso la muerte ${ }^{44,45}$.

\section{Estrongiloidliasis e infección por VIH}

El VIH es un retrovirus que afecta la inmunidad celular, destruyendo o perjudicando su función. A medida que la infección progresa, la inmunidad se torna más débil, y el individuo se vuelve más susceptible a infecciones oportunistas, - estado de $\mathrm{SIDA}^{48}$. Desde la aparición del VIH/SIDA, los estudios discuten el papel de la infección por el virus como un factor de riesgo para el desarrollo de la estrongiloidiasis y sus formas graves ${ }^{49,50}$. Feitosa y cols., sugieren que $S$. stercoralis produciría problemas intestinales en pacientes infectados con VHH/SIDA, siendo responsable por cuadros de diarrea crónica ${ }^{14}$.

A pesar de estar bien definida en la literatura médica la relación del VIH con las infecciones oportunistas, la asociación con la infección por $S$. stercoralis todavía no está totalmente confirmada, persistiendo dudas sobre este conocimiento ${ }^{15,49}$. Un estudio realizado por Dias y cols., en 2012, en pacientes con y sin infección por VIH, no detectó una diferencia significativa en la prevalencia de la parasitosis observada en los dos grupos ${ }^{51}$. Estudios epidemiológicos que investigaron la ocurrencia de la estrongiloidiasis en pacientes infectados por VIH identificaron frecuencias que variaron desde 2,2 hasta $30,1 \%$ y sugirieron que la prevalencia de la infección por el parásito no posee relación directa con el VIH, sino con el status inmunológico que los pacientes presentan ${ }^{15,52,53}$. 
La introducción de la terapia anti-retroviral en 1996 representó un gran avance en el cuidado de los pacientes portadores de VIH/SIDA, lo que posibilitó una reducción de la morbi-mortalidad y un cambio significativo en el perfil de la evolución natural de la infección por $\mathrm{VIH}^{54}$. A pesar de esto, los protocolos clínicos actuales todavía preconizan el monitoreo de infecciones oportunistas en este grupo. En este contexto, la infección por $S$. stercoralis también debe ser una preocupación, incluso en aquellos pacientes donde el tratamiento esté adecuadamente ajustado y el paciente presente baja carga viral o indetectable, con niveles de linfocitos T CD4+ superiores a 400 céls $/ \mathrm{mm}^{3}$.

\section{Estrongilloidiasis e infección por HTLV-1}

La leucemia/linfoma de células T del adulto (LLTA) es una enfermedad asociada al HTLV-1, existiendo cerca de 20 millones de portadores de este virus alrededor del mundo ${ }^{55}$. De todas las condiciones causantes de inmunosupresión, relacionadas a estrongiloidiasis, la infección por HTLV-1 ha sido una de la más fuertemente asociada a las formas graves de la parasitosis ${ }^{56}$. En regiones donde ambos agentes son endémicos (S. stercoralis y HTLV-1) el desarrollo de estrongiloidiasis grave es más frecuente, debido a que este virus provoca una reducción en la producción de los componentes inmunológicos que participan en los mecanismos de defensa contra el parásito ${ }^{57}$.

La asociación entre HTLV-1 y la estrongiloidiasis todavía no está bien dilucidada, siendo imposible definir con precisión cuál es el papel de cada uno de los agentes sobre la patogenia y las complicaciones clínicas del otro ${ }^{58}$. Un estudio publicado por Matutes indica que individuos portadores de HTLV-1 presentan una mayor predisposición al desarrollo de infecciones oportunistas y sus formas graves, entre ellas la producida por S. stercoralis; ese fenómeno ha sido atribuido al compromiso de la inmunidad celular $^{59}$. Otra teoría, igualmente discutida en la literatura científica, indica lo contrario, sugiriendo que la infección por Strongyloides induciría un desarrollo más precoz de LLTA en aquellos pacientes portadores de HTLV $-1^{60}$.

En la literatura médica, diversos estudios relatan la frecuencia de la co-infección HTLV-1/Strongyloides. Para Gotuzzo y cols., la incidencia de HTLV-1 en pacientes con estrongiloidiasis fue de $85,7 \%$ (18/21) contra 4,7\% $(1 / 21)$ en el grupo control ${ }^{58}$. Chieffi y cols., identificaron frecuencias de estrongiloidiasis de 12,1\% (11/91) en individuos infectados por el HTLV-1 y de apenas 1,6\% (1/61) en individuos no portadores del virus ${ }^{60}$. Aguiar demostró que la seroprevalencia de anticuerpos anti- HTLV-1 y anti- HTLV-2 fue menor en pacientes con estrongiloidiasis $(5,5 \%)$ cuando se compararon con individuos no infectados por el virus $(94,5 \%)$; se evidenció una mayor susceptibilidad a la infección por el parásito en individuos infectados por HTLV $-1^{57}$.
En la práctica clínica, el monitoreo de la estrongiloidiasis en pacientes portadores de HTLV-1, y viceversa, es de extrema importancia para viabilizar un mejor pronóstico del paciente, reduciendo la posibilidad de surgimiento de formas complicadas de ambas infecciones ${ }^{54,62}$.

\section{Tratamiento}

El tratamiento de la estrongiloidiasis es complejo porque, a diferencia de lo que ocurre con otras infecciones helmínticas, la erradicación completa es difícil de asegurar debido a la baja carga de parásitos y a la producción irregular de larvas. No se puede afirmar una cura verdadera sólo en base al examen negativo de materias fecales de control ${ }^{63}$.

Para el tratamiento de la estrongiloidiasis es recomendable el uso de ivermectina $200 \mathrm{mg} / \mathrm{kg} /$ día por vía oral durante dos días; debiendo repetirlo en 14 días ${ }^{63}$. En pacientes inmunocomprometidos deben realizarse tres ciclos de tratamiento con intervalos de 14 días entre cada ciclo $^{64,65}$.

Una alternativa es la utilización de tiabendazol $25 \mathrm{mg} /$ $\mathrm{kg}$ dos veces al día, durante 3 a 10 días $^{66}$. Sin embargo, el tratamiento con ivermectina ha sido mejor tolerado por los pacientes ${ }^{65}$.

\section{Profilaxis}

Hasta el momento, no existe régimen quimioprofiláctico adecuado alguno ni tampoco vacunas para la estrongiloidiasis. Sin embargo, algunos cuidados pueden auxiliar en la prevención de la parasitosis; por ejemplo, acciones de saneamiento básico como eliminación adecuada de excretas y aporte de agua potable, medidas higiénicas de lavado de manos después del uso del baño y antes de la manipulación de alimentos, desinfección de alimentos antes del consumo y uso de calzados al caminar por áreas en las que exista la posibilidad de que el suelo esté infectado ${ }^{63,64}$.

\section{Conclusiones}

Las enfermedades tropicales olvidadas comprenden un conjunto de enfermedades de alta importancia, no solamente desde el punto de vista de salud pública sino también económica, ya que acarrea pérdidas económicas significativas para los gobiernos. Dentro de este contexto, se debe dar especial atención a la estrongiloidiasis, principalmente en áreas tropicales y subtropicales, donde el parásito es endémico, por el hecho de ser una parasitosis silenciosa en la mayoría de las veces y que si no es tratada puede evolucionar a formas graves e inclusive la muerte. 


\section{Infectología al Día}

En pacientes inmunocomprometidos, la asociación todavía no está claramente dilucidada y por eso se requiere la realización de nuevos estudios. Sin embargo es consenso en la literatura científica que los individuos que presenten alguna condición que curse con reducción de la respuesta inmune deben ser vigilados debido a la posible co-infección por S. stercoralis. Este monitoreo tiene como objetivo disminuir la morbi-mortalidad producida por la estrongiloidiasis, a través de un diagnóstico precoz y tratamiento medicamentoso adecuado.

Cabe resaltar que siendo la estrongiloidiasis una geo-helmintiasis, su prevención y control necesita de la adopción de un conjunto complejo de acciones que integren estado, equipo de salud y población, basadas en la implementación de saneamiento básico, agua potable y en la promoción de la educación en salud en todos los ámbitos públicos, con el fin de promover un ambiente saludable.

\section{Resumen}

La estrongiloidiasis es una parasitosis que representa un grave problema de salud pública, principalmente en países ubicados en regiones tropicales y subtropicales. A lo largo de los años, algunas condiciones, como por ejemplo, avances en el tratamiento con corticosteroides y enfermedades que evolucionan con inmunosupresión, han favorecido no solamente al aumento de casos de estrongiloidiasis, sino también al surgimiento de formas graves de la enfermedad y/u decesos. Por lo expuesto, el objetivo del presente estudio fue realizar un análisis crítico de la ocurrencia de la estrongiloidiasis en portadores de co-morbilidades, describiendo las características clínico-epidemiológicas de esa asociación que puedan resaltar la importancia de vigilar esta parasitosis en grupos considerados más susceptibles.

\section{Referencias bibliográficas}

1.- World Health Organization. Preventive chemotherapy in human helminthiasis. Coordinated use of anthelmintic drugs in control interventions: a manual for health professionals and programme managers. Geneva: WHO; 2006. http://apps.who.int/iris/ bitstream/10665/43545/1/9241547103 eng.pdf

2.- World Health Organization. Soil-transmitted helminth infections. WHO Fact Sheet $\mathrm{N}^{\circ} 366$. Geneva: WHO; 2015.

3.- Ferreira M S, Nishioka S A, Borges A S, Costa-Cruz J M, Rossin I R, Rocha A, et al. Strongyloidiasis and infection due to human immunodeficiency virus: 25 cases at a Brazilian teaching hospital, including seven cases of hyperinfection syndrome. Clin Infect Dis 1999; 28: 154-5.

4.- Bisoffi Z, Buonfrate D, Montresor A, RequenaMéndez A, Muñoz J, Krolewiecki A J, et al. Strongyloides stercoralis: a plea for action. PLoS Negl Trop Dis 2013; 7: e2214.

5.- Organização Mundial de Saúde. Strongyloidiasis. Disponível em: $<$ http:// www.who.int/intestinal_worms/epidemiology/ strongyloidiasis/en/> acesso em: 19/01/2016.

6.- Paula F M, Costa-Cruz J M. Epidemiological aspects of strongyloidiasis in Brazil. Parasitol 2011; 138: 1331-40.

7.- Luna O B, Gresselli R, Ananias M, Pinto T S, Bozza F A, Soares M, et al. Estrongiloidíase disseminada: diagnóstico e tratamento. RBTI 2007; 19: 463 .

8.- Gottardi M. Imunodiagnóstico da infecção por Strongyloides stercoralis em pacientes candidatos a transplante, São Paulo, Faculdade de Ciências Médicas, Universidade de São Paulo. [Dissertação de Mestrado]. 2014.

9.- Vadlamudi R S, Chi D S, Krishnaswamy G. Intestinal strongyloidiasis and hyperinfection syndrome. Clin Mol Allergy 2006; 30: 4-8.

10.- Newberry A M, Williams D N, Stauffer W M, Boulware D R, Hendel-Paterson B R, Walker P F. Strongyloides hyperinfection presenting as acute respiratory failure and gram-negative sepsis. Chest 2005; 128: 3681-4.

11.- Concha R d, Harrington W J, Rogers A I. Intestinal strongyloidiasis. Recognition, management, and determinants of outcome. $\mathrm{J}$ Clin Gastroenterol 2005; 39: 203-11.

12.- Foreman E, Abraham P J, Garland J L. Not your typical strongyloides infection: a literature review and case study. South Med J 2006; 99; 847-52.

13.- Fardet L, Genereau T, Poirot J L, Guidte B, Kettaneh A, Cabane J. Severe strongyloidiasis in corticosteroid-treated patients: case series and literature review. J infect 2007; 54: 18-27.

14.- Feitosa G, Bandeira C, Sampaio D P, Badaró R, Brites C. High prevalence of giardiasis and strongyloidiasis among HIV-infected patients in Bahia, Brazil. The Brazilian strongyoloidiasis in Brazil. J infect Dis 2001; 5: 339-44.

15.- Getaneh A, Medhin G, Shimelis T. Cryptosporidium and Strongyloides stercoralis infections among people with and without HIV infection and efficiency of diagnostic methods for Strongyloides in Yirgalem Hospital, southern, Ethiopia. BMC Res Notes 2010; 3: 90.

16.- Schaffel R, Nucci M, Carvalho E, Braga M, Almeida L, Portugal R, et al. The value of immunoenzymatic test (enzyme-linked immunosorbent assay) for the diagnosis of strongyloidiasis in patients immunosuppresed by hematologic malignancies. Am J Trop Med Hyg 2001; 65: 346-50.

17.- Graeff-Teixeira C, Leite C S M, Sperhacke C L, Fassina K, Petry S M G, Mucenic T, et al. Prospective study of strongyloidiasis in patients with hematologic malignancies. Rev Soc Bra Med Trop 1997; 30: 355-7.

18.- Martín-Dávila P, Fortún J, López-Vélez R, Norman F, Montes de Oca M, Zamarrón P, et al.

Transmission of tropical and geographically restricted infections during solid-organ transplantation. Clin Microbiol Rev 2008; 21: 60-96.

19.- Kotton C N. Zoonoses in solid-organ and hematopoietic stem cell transplant recipients. Clin Infec Dis 2007; 44: 857-66.

20.- Mani B C, Mathur M, Clauss H, Álvarez R, Amad E, Toyoda Y, et al. Strongyloides stercoralis and organ transplantation. Case Reports in Transplantation, Hindawi 2013; 1: 1-6.

21.- Marcos L A, Terashima A, Canales M, Gotuzzo E. Update on strongyloidiasis in the immunocompromised host. Curr Infect Dis Rep 2011; 13: 35-46.

22.- Batista M V, Pierrotti L C, Abdala E, Clemente W T, Girão E S, Rosa D R, et al. Endemic and opportunistic infections in brasilian solid organ transplant recipients. Trop Med Int Health 2011; 16: 1134-42.

23.- Coster L O. Parasitic infections in solid organ transplant recipients. Infect Dis Clin North Am 2013; 27: 395-427.

24.- Franco-Pareces C, Jacob J T, Hidron A, 
Rodríguez-Morales J A, Kuhar D, Caliendo A M. Transplantation and tropical infectious diseases. Int J Infect Dis 2010; 14: 189-96.

25.- Fitzpatrick M A, Caicedo J C, Stosor V, Ison M G. Expanded infectious diseases screening program for Hispanic transplant candidates. Transplantation and tropical infectious diseases. Int J Infect Dis 2010; 12: 336-41.

26.- Palau L A, Pankey G A. Strongyloides hyperinfection in a renal transplant recipient receiving cyclosporine: possible Strongyloides stercoralis transmission by kidney transplant. Am J Trop Med Hyg 1997; 57: 413-5.

27.- Vilela E G, Clemente W T, Mira R R L, Torres H O G, Veloso L F, Fonseca L P, et al. Strongyloides stercoralis hyperinfection syndrome after liver transplantation: case report and literature review. Transpl Infect Dis 2009; 11: 132-36.

28.- Schaeffer M W, Buell J F, Gupta M, Conway G D, Akhter S A, Wagoner L E. Strongyloides hyperinfection syndrome after heart transplantation: case report and review of the literature. J Heart Lung Transpl 2004; 23 : 905-11.

29.- Orlent H, Crawley C, Cwynarski K, Dina R, Apperley J. Strongyloidiasis pre and post autologous peripheral blood stem cell transplantation. Bone Marrow Transplant 2003; 32: $115-7$

30.- Oliveira L C, Ribeiro C T, Mendes D M, Oliveira T C, Costa-Cruz J M. Frequency of Strongyloides stercoralis infection in alcoholics. Mem Inst Oswaldo Cruz 2002; 97 : 119-21.

31.- Zago-Gomes M P, Aikawa K F, Perazzio S F, Gonçalves C S, Pereira F E. Prevalence of intestinal nematodes in alcoholic patients. Rev Soc Bras Med Trop 2002; 35: 571-4.

32.- Marques C C, Zago-Gomes M P, Gonçalves S C S, Pereira F E L. Alcoholism and Strongyloides stercoralis: Daily ethanol ingestion has a positive correlation with the frequency of Strongyloides larvae in the stools. Negl Trop Dis 2010; 4: 717-21.

33.- Silva M L S, Inêsa E J, Souza A B S, Dias V M S, Guimarães C M, Menezes E R, et al. Association between Strongyloides stercoralis infection and cortisol secretion in alcoholic patients. Acta Trop 2016; 154: 133-8.

34.- Gaburri D, Gaburri K, Hubner E, Lopes M H, Ribeiro A M, Paulo G A, et al. Intestinal parasitosis and hepatic cirrhosis. Arq Gastroenterol 1997; 34: 7-12.

35.- Bona S, Basso R M C. Hyperinfection by Strongyloides stercoralis associated with chronic use of corticosteroid. Rev Bras Anal Clin 2008; 40: 247-50.

36.- Concha R, Harrington W J R, Rogers A I.
Intestinal strongyloidiasis: Recognition, management, and determinants of outcome. J Clin Gastroenterol 2005; 39: 203-11.

37.- Addolorato G, Montalto M, Capristo E, Certo M, Fedeli G, Gentiloni N, et al. Influence of alcohol on gastrointestinal motility lactulose breath hydrogen testing in oro-cecal transit time in chronic alcoholics, social drinkers and teetotaler subjects. Hepato-Gastroenterol 1997; 44: 1076-81.

38.- Teixeira M C, Inês E J, Pacheco F T, Silva R K, Mendes A V, Adorno E V, et al. Asymptomatic Strongyloides stercoralis hyperinfection in an alcoholic patient with intense anemia. J Parasitol 2010; 96: 833-5.

39.- Sociedade Brasileira de Endocrinologia e Metabologia. O que é Diabetes? Disponível $\mathrm{em}:<\mathrm{http}: / /$ www.endocrino.org.br/o-que-ediabetes/> Acesso em: 21/02/2016.

40.- Mendonça S C, Gonçalves-Pires M R F, Rodrigues R M, Ferreira A J R, Costa-Cruz $\mathrm{J} M$. Is there an association between positive Strongyloides stercoralis serology and diabetes mellitus? Acta Trop 2006; 99: 102-5.

41.- Nazligul Y, Sabuncu T, Ozbilge H. Is there a predisposition to intestinal parasitosis in diabetic patients? Diabetes Care 2001; 24: 1503-4.

42.- Bessman A N, Sapico F L. Infections in the diabetic patient: the role of immune dysfunction and pathogen virulence factors. J Diabetes Complications 1992; 6: 258-62.

43.- Instituto do Câncer. Câncer de estômago. Disponível em: $<$ http://www2.inca.gov.br/ wps/wcm/connect/tiposdecancer/site/home/ estomago/definicao > Acesso em: 20/02/2016

44.- Machado E R, Teixeira E M, Gonçalves-Pires Mdo R, Loureiro Z M, Araújo R A, CostaCruz J M. Parasitological and immunological diagnosis of Strongyloides stercoralis in patients with gastrointestinal cancer. Scand J Infect Dis 2008; 40: 154-8.

45.- Safdar A, Malathum K, Rodríguez S J, Husni R, Rolston K V I. Strongyloidiasis in patients at a Comprehensive Cancer Center in the United States. Am Cancer Soc 2004; 100: 1531-6.

46.- Scowden E B, Schaffner W, Stone W J. Overwhelming strongyloidasis: an unappreciated opportunistic infection. Med (Baltimore) 1978; 57: 527-44.

47.- Madbouly K M, Senagore A J, Mukerjee A, Hussien A M, Shehata M A, Navine P, et al. Colorectal cancer in a population with endemic Schistosoma mansoni: is this an at-risk population? Int J Colorectal Dis 2007; 22: 175-81.

48.- Organização Mundial de Saúde. Proyecto de estrategia 2016-2021: VIH/sida, hepatitis víricas e infecciones de transmisión sexual. Disponível em: <http:// http://www.who.int/hiv/ strategy2016-2021/es/> acesso em: 12/01/2016.

49.- Eyal N, Oren Z. Severe strongyloidiasis in AIDS: relative risk obscured by absolute rarity. AIDS 2016, 30: 671-76.

50.- Siddiqui U, Bini E J, Chandarana K, Leong J, Ramsetty S, Schiliro D, et al. Prevalence and impact of diarrhea on health-related quality of life in HIV-infected patients in the era of highly active antiretroviral therapy. J Clin Gastroenterol 2007; 41: 484-90.

51.- Dias R M D S, Mangini A C S, Torres D M A G V, Vellosa S A G, Silva M I P G, Silva R M $\mathrm{S}$, et al. Ocorrência de Strongyloides stercoralis em pacientes portadores da síndrome de imunodeficiência adquirida (AIDS). Rev Inst Med Trop São Paulo 1992; 34: 15-7.

52.- Bachur T P R, Vale J M, Coêlho I C B, De Queiroz T R B S, Chaves C S. Enteric parasitic infections in HIV/AIDS patients before and after the highly active antiretroviral therapy. Braz J Infect Dis 2008; 12: 115-22.

53.- Blatt J M, Cantos G A. Evaluation of techniques for the diagnosis of Strongyloides stercoralis in human immunodeficiency virus (HIV) positive and HIV negative individuals in the city of Itajaí, Brazil. Braz J Infect Dis 2003; 7: 402-8.

54.- Willemot P, Klein M B. Prevention of HIVassociated opportunistic infections and diseases in the age of highly active antiretroviral therapy. Expert Rev Anti Infect Ther 2004; 2 : 521-32.

55.- Lage L A P C, Costa R O, Mariano L C B, Xavier E M, Pereira J. Resistant and disseminated Strongyloides stercoralis infection in a young patient with adult T-cell leukemia/ lymphoma. Rev Bras Hematol Hemoter 2012; 34: 464-6.

56.- Porto M A F, Muniz A, Oliveira-Jr J, Carvalho E M. Implicações clínicas e imunológicas da associação entre o HTLV I e a estrongiloidíase. Rev Soc Bras Med Trop 2002; 35: 641-49.

57.- Aguiar S A. Prevalencia de HTLV-1 E HTLV-2 em portadores de Strongyloides stercoralis, Pará, Brasil. Programa de Pós-Graduação em Doenças Tropicais. Universidade Federal do Pará. [Dissertação de Mestrado]. 2012.

58.- Gotuzzo E, Arango C, De Queiroz-Campos A, Isturiz R E. Human T-cell lymphotropic virus-I in Latin America. Infect Dis Clin North Am 2000; 14: 211-39.

59.- Matutes E. Adult T-cell leukemia-lymphoma. J Clin Pathol 2007; 60: 1373-7.

60.- Plumelle Y, Gonin C, Edouard A, Bucher B J, Thomas L, Brabion A, et al. Effect of Strongyloides stercoralis infection and eosinophilia on age at onset and prognosis of adult T-cell leukemia. Amer J Clin Pathol 1997; 107: 81-7.

61.- Chieffi P P, Chiattone C S, Feltrim E M, 
Alves R C, Paschoalotti M A. Coinfection by Strongyloides stercoralis in blood donors infected with human T-cell leukemia/lynphoma virus type 1 in São Paulo city, Brazil. Mem Inst Oswaldo Cruz 2000; 95: 711-2.

62.- Carvalho E M, Da Fonseca P A.

Epidemiological and clinical interaction between HTLV-1 and Strongyloides stercoralis. Parasite Immunol 2004; 26: 487-97.
63.- World Gastroenterology Organisation. Practice Guidelines: Manejo de la Estrongiloidiasis. Disponível em: $<$ http:// www.worldgastroenterology.org/UserFiles/file/ guidelines/management-of-strongyloidiasisspanish.pdf> acesso em: 01/12/2016.

64.- Corti M. Review article: Strongyloides stercoralis in immunosuppressed patients. Arch Clin Infect Dis 2016; 11: e27510.
65.- Zhang R Y, Chen B J, Lin J X. Ivermectin in intractable Strongyloides stercoralis infection. Strait J Prevent Med 2002; 8: 79.

66.- Braz A S, Andrade C A F, Mota L M H, Lima C M B L. Recommendations from the Brazilian Society of Rheumatology on the diagnosis and treatment of intestinal parasitic infections in patients with autoimmune rheumatic disorders. Rev Bras Reumatol 2015; 55: 368-80. 